\title{
MULTI-TARGET DETECTION FROM FULL-WAVEFORM AIRBORNE LASER SCANNER USING PHD FILTER
}

\author{
T. Fuse ${ }^{\text {a }}$, D. Hiramatsu ${ }^{\text {a }}$, W. Nakanishi ${ }^{\text {a, } *}$ \\ ${ }^{a}$ Dept. of Civil Engineering, The University of Tokyo, 7-3-1 Hongo, Bunkyo-ku, Tokyo 1138656 Japan \\ - (fuse@civil, hiramatsu@trip, nakanishi@civil).t.u-tokyo.ac.jp
}

Commission V, WG V/4

KEY WORDS: Probability hypothesis density, Full-waveform airborne laser scanner, Multi-target detection

\begin{abstract}
:
We propose a new technique to detect multiple targets from full-waveform airborne laser scanner. We introduce probability hypothesis density (PHD) filter, a type of Bayesian filtering, by which we can estimate the number of targets and their positions simultaneously. PHD filter overcomes some limitations of conventional Gaussian decomposition method; PHD filter doesn't require a priori knowledge on the number of targets, assumption of parametric form of the intensity distribution. In addition, it can take a similarity between successive irradiations into account by modelling relative positions of the same targets spatially. Firstly we explain PHD filter and particle filter implementation to it. Secondly we formulate the multi-target detection problem on PHD filter by modelling components and parameters within it. At last we conducted the experiment on real data of forest and vegetation, and confirmed its ability and accuracy.
\end{abstract}

\section{INTRODUCTION}

Full-waveform airborne laser scanner becomes widely used in LiDAR. Reflection intensity data acquired by full-waveform airborne laser scanner is expected to be useful compared with traditional discrete-return laser system, especially in the case multiple target exist above ground. For example, to retrieve 3D canopy structure from LiDAR data is important with regard to carbon cycle modelling (Hurtt et al., 2004). Many researchers have used Gaussian decomposition to model and analyse fullwaveform data, and detect multiple targets including ground (e.g. Persson et al., 2005; Wanger et al., 2006; Pirotti, 2011). However it is difficult to detect multi-target precisely when reflection intensity from target is weak and not distinguishable from noise. Richter et al. (2014) has proposed the method to correct attenuation reflected by target which are further from scanner. Nevertheless, the two shortcomings with Gaussian decomposition are the following; we have to fix the number of targets in advance, and the reflection intensity might not fit Gaussian distribution.

As discussed in Kleinherenbrink et al. (2014), the reflection intensity of successive signals can be helpful in specifying targets, for the signals are continuous spatially and temporally. Based on this concept of similarity in reflection intensity distribution of neighbourhood signals, we regard the multitarget detection problem as the time-series analysis. We propose a new method of analysing the full-waveform data for multitarget detection by employing probability hypothesis density (PHD) filter. PHD filter is a type of Bayesian filter; Bayesian update system of PHD introduced by Mahler (Mahler, 2000; Mahler, 2003). PHD filter is applied to multi-target tracking, for it can deal with unknown and variable number of objects. More specifically we can estimate the number of targets within the area and their positions simultaneously by PHD filter. In fact, many applications of PHD filter to multi-target tracking have shown that the filter is a robust solution to deal with uncertain factors such as number of targets and accuracy of observation (e.g. Tobias and Lanterman, 2004; Ikoma et al., 2008).

Our idea is that the problem of variable-number multi-target tracking by still sensor is similar to the problem of variablenumber multi-target detection by moving sensor, e.g. aerial laser scanner. The structure of these problems are almost the same; what we can observe are generated stochastically by latent variables, or "state", and state follows a certain dynamics and it sometimes appears and disappears. What generates timeseries changing is the only difference; in tracking, motion of target does, and in detection, irradiation angle of sensor. In the following section, we explain PHD filter, formulate multi-target detection problem by using it, and then apply a proposed method to the acquired data of forest and vegetation.

\section{PROBABILITY HYPOTHESIS DENSITY FILTER}

\subsection{Sequential Bayesian Filtering}

Consider a general state space model (Figure 1): non-Gaussian and nonlinear state space model for time series $\mathbf{z}_{k}$,

$$
\begin{aligned}
& \mathbf{x}_{k} \sim p\left(\mathbf{x}_{k} \mid \mathbf{x}_{k-1}\right) \\
& \mathbf{z}_{k} \sim p\left(\mathbf{z}_{k} \mid \mathbf{x}_{k}\right)
\end{aligned}
$$

where $\mathbf{x}_{k}$ is an unknown state vector and the conditional probability functions of each equation are not necessarily Gaussian. Equation (1) is called the system model and equation (2) the observation model. After we obtain $\mathbf{z}_{1: k}=\left\{\mathbf{z}_{1}, \mathbf{z}_{2}, \ldots, \mathbf{z}_{k}\right\}$,

\footnotetext{
* Corresponding author
} 
a series of observations from time 1 to $k$, the posterior distribution of $\mathbf{x}_{k}$ is calculated by Bayes' theorem as follows:

$$
\begin{aligned}
& p\left(\mathbf{x}_{k} \mid \mathbf{z}_{1: k}\right) \propto p\left(\mathbf{z}_{k} \mid \mathbf{x}_{k}\right) p\left(\mathbf{x}_{t} \mid \mathbf{z}_{1: k-1}\right) \\
& \quad=p\left(\mathbf{z}_{k} \mid \mathbf{x}_{k}\right) \int p\left(\mathbf{x}_{k} \mid \mathbf{x}_{k-1}\right) p\left(\mathbf{x}_{k-1} \mid \mathbf{z}_{1: k-1}\right) d \mathbf{x}_{k-1}
\end{aligned}
$$

Equation (3) shows the process of sequential Bayesian filtering. It includes the system model and the observation model, and the estimated result at time $k-1: p\left(\mathbf{x}_{k-1} \mid \mathbf{z}_{1: k-1}\right)$. Therefore we can sequentially estimate the posterior of $\mathbf{x}_{k}$ by this equation. If we need the estimated value for $\mathbf{x}_{t}$, an expected value of $p\left(\mathbf{z}_{t} \mid \mathbf{x}_{t}\right)$ is usually used.

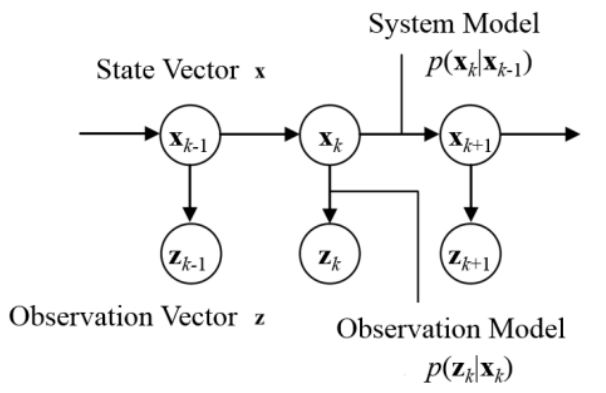

Figure 1. General state space model

\subsection{PHD Filter}

Equation (3) can be directly applied to the single- or constantnumber-target detection. However, considering a variablenumber multi-target detection, the dimension of $\mathbf{x}_{k}$ should increase or decrease appropriately according to the increase or the decrease of targets. Thus equation (3) cannot be directly used for problems in such situations, which are indeed true with real application.

Consider a random and non-negative integer $n(k)$, the number of targets at time $k$, and a state vector $\mathbf{X}_{k}=\left\{\mathbf{x}_{1}, \mathbf{x}_{2}, \ldots, \mathbf{x}_{n(k)}\right\}$ at time $k$. As $n(k)$ is stochastic, $\mathbf{X}_{k}$ is also a random variable (vector). Usually state space of $\mathbf{X}_{k}$ is not computable because its dimension increases exponentially according to the number of objects. Instead, probability hypothesis density (PHD), the first order moment of this state space is proposed. And PHD is shown to be identical to the factorial moment density in point process theory, so PHD can be any density function that when integrated over the interested area, the integration of it is the expected number of the targets within that area. Therefore taking multi-target detection as an example, under a set of timeseries observations $\mathbf{Z}_{1: k}=\left\{\mathbf{Z}_{1}, \mathbf{Z}_{2}, \ldots, \mathbf{Z}_{k}\right\}$ from sensors, we can estimate the number of targets $n(k)$ and their positon $\mathbf{x}_{k}$ at time $k: \mathbf{X}_{k}=\left\{\mathbf{x}_{1}, \mathbf{x}_{2}, \ldots, \mathbf{x}_{n(k)}\right\}$ by PHD filter.

We indicate PHD of a state vector $\mathbf{x}$ as $D(\mathbf{x})$. Then the predictive PHD at time $k$ given observation from time 1 to $k-1$ is described as

$$
D\left(\mathbf{x}_{k} \mid \mathbf{Z}_{1: k-1}\right)=p_{s} \int p\left(\mathbf{x}_{k} \mid \mathbf{x}_{k-1}\right) D\left(\mathbf{x}_{k-1} \mid \mathbf{Z}_{1: k-1}\right) d \mathbf{x}_{k-1}+D_{\text {birth }}\left(\mathbf{x}_{k}\right)
$$

where $p_{s}$ is the survival probability that the target $\mathbf{x}$ at time $k$-1 will appear at $k, D_{\text {birth }}\left(\mathbf{x}_{k}\right)$ is the PHD of the likelihood function that makes a new targets $\mathbf{x}$ at time $k$, and $p\left(\mathbf{x}_{k} \mid \mathbf{x}_{k-1}\right)$ is the singletarget motion model: system model of equation (1). Then the posterior of PHD after observation at time $k$ is that

$$
\begin{aligned}
& D\left(\mathbf{x}_{k} \mid \mathbf{Z}_{1: k}\right) \\
& =\left\{\left(1-p_{d}\right)+p_{d} \sum_{\mathbf{z} \in \mathbf{Z}_{k}} \frac{p(\mathbf{z} \mid \mathbf{x})}{\mu_{c} p_{c}(\mathbf{z})+p_{d}\left\langle p(z \mid \cdot), D\left(\cdot \mid \mathbf{Z}_{1: k-1}\right)\right\rangle}\right\} D\left(\mathbf{x}_{k} \mid \mathbf{Z}_{1: k-1}\right)
\end{aligned}
$$

where $p_{d}$ is (positive, both true and false) detection rate of $\mathbf{x}, \mu_{c}$ is the expected value of false detection, $p_{c}(\mathbf{z})$ is false detection distribution, $\langle f(\cdot), g(\cdot)\rangle$ denotes convolution of functions $f$ and $g$ with respect to $\cdot$, and $p(\mathbf{z} \mid \mathbf{x})$ is the single-target observation model of equation (2): the conditional probability density of observation $\mathbf{z}$ given $\mathbf{x}$.

The integration of posterior of PHD (equation (5)) is the expectation of the number of targets at time $k, n(k)$. That is,

$$
E[n(k)]=\int D\left(\mathbf{x}_{k} \mid \mathbf{Z}_{1: k}\right) d \mathbf{x}_{k}
$$

and usually we use the nearest integer of $\mathrm{E}[n(k)]$ as the estimated value of number of targets at time $k$.

\subsection{Particle Filter Implementation of PHD Filter}

In this subsection, we explain how to calculate equation (4) and (5) using particle approximation (Figure 3). This implementation has been proposed by some researchers (Vo et al., 2003; Zajic and Mahler, 2003; Vo et al., 2005).

\subsubsection{Preparation: Consider a set of particles}

$$
\left\{\left(\mathbf{x}_{k-1, i}, w_{i}\right)\right\}_{i=1}^{L_{k-1}}
$$

that approximates the PHD at time instance $k-1$, where $\mathbf{x}_{k-1, i}$ is a realisation of $\mathrm{D}\left(\mathbf{x}_{k-1}\right), w_{i}$ is the weight of particle $i$, and $L_{k-1}$ is the number of particles and at time $k-1$. Set the number of particles $\rho$ par one object. Then $L_{k-1}=\rho \times \mathrm{E}[n(k-1)]$ and $w_{i}=1 / \rho$.

2.3.2 System Model: Firstly calculate the first term of righthand member of equation (4) by set $w_{i}=p_{s} / \rho$ :

$$
\begin{aligned}
& p_{s} \int p\left(\mathbf{x}_{k} \mid \mathbf{x}_{k-1}\right) D\left(\mathbf{x}_{k-1} \mid \mathbf{Z}_{1: k-1}\right) d \mathbf{x}_{k-1} \simeq\left\{\left(\mathbf{x}_{k, i}, p_{s} w_{i}\right)\right\}_{i=1}^{L_{k-1}} \\
& \mathbf{x}_{k, i} \sim p\left(\mathbf{x}_{k} \mid \mathbf{x}_{k-1}=\mathbf{x}_{k-1, i}\right)
\end{aligned}
$$

This calculation corresponds to the survival of the target. Then calculate the second term of right-hand member of equation (4) by generate $\rho$ particles drawn from $D_{\text {birth }}\left(\mathbf{x}_{k}\right)$ with their weight $\mu_{\text {birth }} / \rho$, where $\mu_{\text {birth }}$ is the expected number of newly generated object defined by $D_{\text {birth }}\left(\mathbf{x}_{k}\right)$.

2.3.3 Observation Model: At this point the number of particles is $L_{k-1}+\mu_{b i r t h} / \rho$. For each particle $i=1,2, \ldots, L_{k-1}+$ $\mu$ birth $\rho$, calculate and update

$$
w_{i} \leftarrow\left\{\left(1-p_{d}\right)+p_{d} \sum_{\mathbf{z} \in \mathbf{Z}_{k}} \frac{p\left(\mathbf{z} \mid \mathbf{x}=\mathbf{x}_{i}\right)}{\mu_{c} p_{c}(\mathbf{z})+\sum_{i} w_{i} p\left(\mathbf{z} \mid \mathbf{x}=\mathbf{x}_{i}\right)}\right\} w_{i}{ }^{(9)}
$$

Then we obtain the particle approximation of posterior PHD at time instance $k$ as

$$
\left\{\left(\mathbf{x}_{k, i}, w_{i}\right)\right\}_{i=1}^{L_{k-1}+\mu_{\text {birth }} / \rho}
$$


2.3.4 Estimation and Resampling: Calculate equation (6) as

$$
E[n(k)] \simeq \sum_{i} w_{i}
$$

and the number of particles to be resampled as

$$
L_{k}=\rho \sum_{i} w_{i}
$$

Then resample particles by drawing $L_{k}$ times from the posterior PHD: the probability of each particle proportional to its weight. Now we obtain equation (7) at time $k$, and then repeat from 2.3.2.

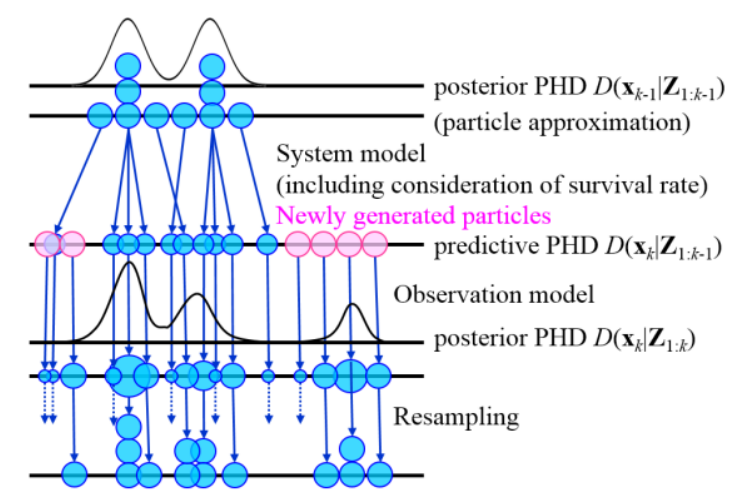

Figure 2. Particle filter implementation of PHD filter

\section{PHD FILTERING FORMULATION FOR MULTI- TARGET DETECTION}

In this section we formulate the multi-target detection problem using PHD filter.

\subsection{Observation}

Firstly we explain the data and observations. Full-waveform laser scanner records the reflection intensity as a function of elapsed time, or time of flight, $t$ from the laser irradiation at time $k$ (Figure 3). A peak of high intensity implies that there is a target (Figure 4). And we can calculate the distance between the scanner and each reflection point using the speed of light $c$, the relative position using the irradiation angle $\theta$. However there can be a target even if the intensity has only a weak peak. So the problem is how to identify the number of target from the waveform information by relating the peak of intensity to the target and distinguishing them from noise, at each irradiation.

\subsection{Formulation}

3.2.1 State Vector and Observation Vector: We consider a simple situation that we have known the height and attitude of airborne. Also since the scanning rate is high enough, we can assume that the airborne almost remains still and the targets are almost at the same positions in the successive irradiation. Therefore the change of the irradiation angle of the scanner is only considered in the following of this paper. Above these assumptions, we define variable $\mathbf{x}$ as position on ground: $x y$ plane $(x, y)$ and height from scanner $h$. We also define variable $\mathbf{Z}$ as a series of reflection intensity $\mathbf{r}=\left\{r_{1}, r_{2}, \ldots, r_{T}\right\}$.
3.2.2 System Model: If we consider that the dataset is small and thus define $x$ axis parallel to the scanning line (Figure 5), the system model $p\left(\mathbf{x}_{k} \mid \mathbf{x}_{k-1}\right)$ can be written as

$$
p\left(\mathbf{x}_{k} \mid \mathbf{x}_{k-1}\right)=\left(\begin{array}{l}
x_{k}-x_{k-1} \\
y_{k}-y_{k-1} \\
h_{k}-h_{k-1}
\end{array}\right)+\left(\begin{array}{l}
u_{x, k} \\
u_{y, k} \\
u_{h, k}
\end{array}\right)=\left(\begin{array}{l}
x_{k-1} \\
y_{k-1} \\
h_{k-1}
\end{array}\right)+\left(\begin{array}{l}
h_{k-1} \omega \Delta t \\
0 \\
0
\end{array}\right)+\left(\begin{array}{l}
u_{x, k} \\
u_{y, k} \\
u_{h, k}
\end{array}\right)
$$

where $\omega \Delta t=\theta_{k}-\theta_{k-1}(<<1$; quite high scan rate) is the difference of the irradiation angle $\theta$ between time $k$ and $k-1$, and $u$ is random error term for each element of $\mathbf{x}$. The equation that $h_{k}$ is approximately equal to $h_{k-1}$ is based on the high scan rate assumption stated above. And under this assumption, the system model for $x$ is derived as

$$
\begin{aligned}
x_{k}-x_{k-1} & =h_{k} \tan \theta_{k}-h_{k-1} \tan \theta_{k-1} \\
& \simeq h_{k-1} \tan \left(\theta_{k-1}+\omega \Delta t\right)-h_{k-1} \tan \left(\theta_{k-1}\right) \\
& =h_{k-1}\left\{\frac{\tan \theta_{k-1}+\tan (\omega \Delta t)}{1-\tan \theta_{k-1} \tan (\omega \Delta t)}-\tan \theta_{k-1}\right\} \\
& \simeq h_{k-1} \omega \Delta t
\end{aligned}
$$

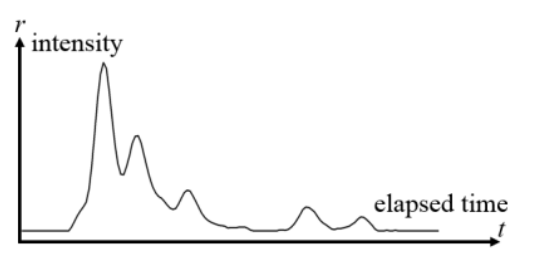

Figure 3. Full-waveform data

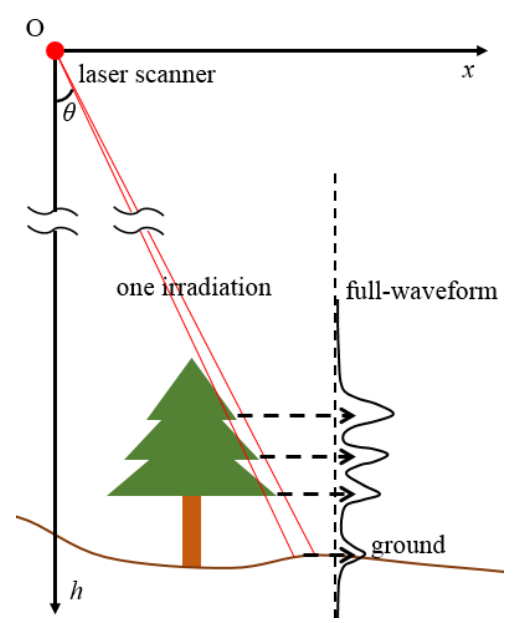

Figure 4. $x$ - $h$ coordinates, reflection intensity and target

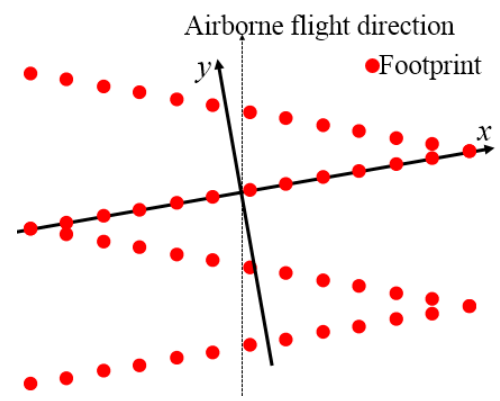

Figure 5. $x-y$ coordinates for small dataset 
3.2.3 Observation Model: We can employ any function that relate the observation $\mathbf{r}$ and the variable $\mathbf{x}$ as an observation model. Note that even in the full-waveform, intensity $r$ is recorded as the convolution of energy in a short time window between $t$ and $t+\Delta t$, and we denote it as the intensity $r_{t}$ at discrete time instance $t$. In this paper, we define the observation model as following model:

$$
p(\mathbf{z} \mid \mathbf{x})=\frac{r_{t^{*}}}{\sum_{t} r_{t}}
$$

where

$$
\begin{aligned}
& t^{*}=\frac{2 \sqrt{x_{k}^{2}+h_{k}^{2}}}{c} \\
& r_{t^{*}}=r_{\left[t^{*}\right\rfloor}\left(\left[t^{*}\right\rceil-t^{*}\right)+r_{\left\lceil t^{*}\right\rceil}\left(t^{*}-\left\lfloor t^{*}\right\rfloor\right)
\end{aligned}
$$

and $c$ is the speed of light. The first equation of (16) means that twice of the distance from the scanner to the target equals the elapsed time $t^{*}$ times $c$, and the second means the interpolation of intensity at $t^{*}$ using neighbour discrete time $t$. This model represents that higher intensity implies higher likelihood of the existence of the target.

3.2.4 Other Variables: For the other components of this PHD filter, we can put the target survival rate $p_{s}$, target birth rate and the distribution for $D_{\text {birth }}\left(\mathbf{x}_{k}\right)$ according to the observation site. Also we can put detection rate $p_{d}$ and false detection $\mu_{c} p_{c}$ according to the observation situation. If we don't have a priori knowledge about them, we usually put constant $p$ and uniformly distributed $D$.

\subsection{Object Identification}

3.3.1 Concept: PHD in equation (4) and (5) doesn't have any information about the target identification. One possible solution to identify each target from PHD is to apply a clustering method at each time. On the other hand, in case of the PHD filter in this paper, we assume that we can track each target before detection at each time $k$, namely "track-beforedetect" approach in the field of visual tracking. In this subsection, we explain the manner to manage labels put on to each target, based on Ikoma et al. (2013). The purpose here is to determine each particle's association with target to calculate the position of each target.

3.3.2 Modification in Filtering: We put a set of positive integer labels on each target. Thus, we add a label variable $l$ to state vector $\mathbf{x}$ of equation (7),

$$
\left\{\left(\mathbf{x}_{k-1, i}, w_{i}, l_{i}\right)\right\}_{i=1}^{L_{k-1}}
$$

and we put $l=0$ for newly generated particles. Then the same as Ikoma et al. (2013), based on $l$ at previous time instance $k$-1, we eliminate other targets from the original reflection signal at time $k$. We calculate state-dependent likelihoods for each target, instead of summation of all targets in equation (9). The estimation result for each target is calculated as the expected value for each label.
3.3.3 Labelling Rule: After we calculate posterior PHD at time $k$, we update the set of labels according to labelling rule. It includes putting new label and removing existing label. We put new positive integer label for particles with $l=0$ if the summation of their weight within a sliding window along $h$ is greater than threshold $l_{\text {add }}$. In the same way, we remove the existing label with positive integer if the summation of their weight are less than threshold $l_{\text {rem }}$, and replace their labels with $l=0$. The threshold $l_{\text {add }}$ and $l_{\text {rem }}$ are defined in advance. Note that this labelling is independent of PHD estimation itself. Thus the integration of particles' weight with a certain label may be different from one. This fact might be strange because the number of one unique target is not necessarily estimated to be one. However, the labelling rule almost works well and the integration usually converges at around one if $l_{\text {add }}$ and $l_{\text {rem }}$ are appropriately controlled.

3.3.4 Initial PHD: At last, we manually set the initial PHD, setting variables $(x, h, l)$ for each target. Although this process can be replaced with automatic target detection method, as we focus on basic performance of PHD filtering step in this paper, we give priority to avoiding any initial errors.

\section{EXPERIMENTS}

\subsection{Data and Setting}

We use the full-waveform data of forest and vegetation at the Institute for Nature Study located in Tokyo. Data was acquired on October 20, 2012, from the altitude of $950 \mathrm{~m}$. Scan rate is $100[\mathrm{kHz}]$, scan rate is $42[\mathrm{~Hz}]$ and scan angle is $30\left[^{\circ}\right]$ (see Nakano and Chikatsu (2015) for more detail). Thus we can calculate $\omega \Delta t=4.4 \times 10^{-4}[\mathrm{rad} / \mathrm{shot}]$ in equation (13) and (14).

Experiment to detect multi-targets in 20 irradiation points that are align is conducted. We define $x$ axis on this alignment so that we can omit the variable $y$ on $x y$-plane. Thus we directly use the system and observation model of equation (13) and (15), respectively. According to the data, we set the parameters shown in Table 6.

\begin{tabular}{|l|c|}
\hline Parameter & Value \\
\hline \# particles par target $\rho$ & 1000 \\
Survival rate $p_{s}$ & 0.7 \\
(*)Target birth (rate) $\mu_{\text {birth }}$ & 0.3 \\
Detection rate $p_{d}$ & 0.9 \\
(*)False detection (rate) $\mu_{c}$ & 0.1 \\
Label putting $l_{\text {add }}$ & 0.4 \\
Label removal $l_{\text {rem }}$ & 0.6 \\
\hline
\end{tabular}

(*)Target birth and false detection rate are assumed to follow a uniform distribution over the state-space in this experiment.

Table 6. Parameter settings

\subsection{Result and Discussion}

Firstly we obtain ground truth data by manually detecting targets from original reflection signals. There are 56 targets during 20 time steps and the number of targets at each time varies from one to four (Figure 7).

Experimental results show that the proposed technique can simultaneously estimate the number of targets and their positions. Figure 7 shows the estimated and true number of targets at each time. Precise estimation is achieved by the 


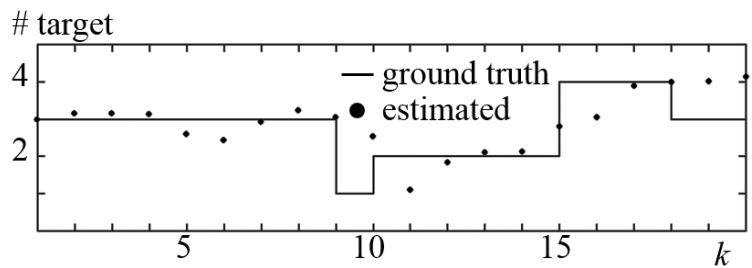

Figure 7. Number of targets at each time $k$

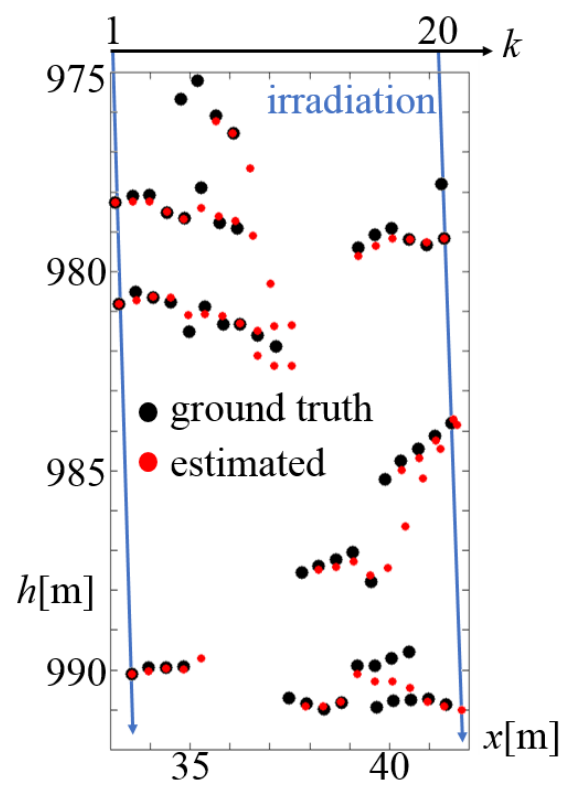

Figure 9. Estimated results on $x$ - $h$ coordinates

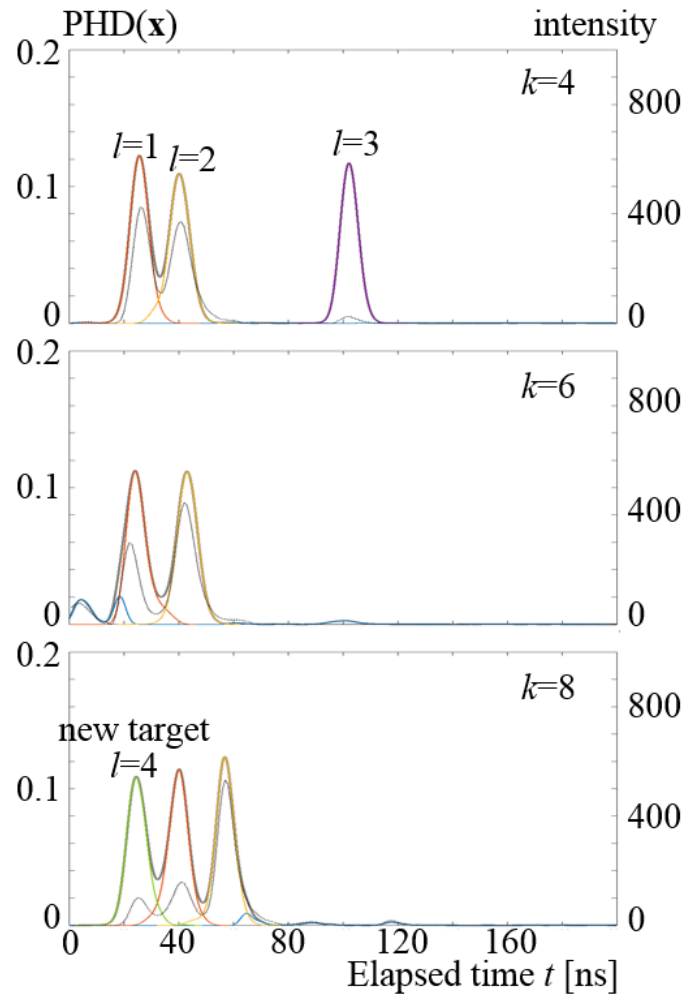

Colour lines show the estimated PHD of each label (left axis) and black shows the signal intensity (right axis).

Elapsed time $t$ can be converted to $x$ - $h$ coordinates by equation (14). Smaller $t$ means smaller $h$ (also see Figure 9)

Figure 8. Estimated PHD

\section{CONCLUSION}

limitation so far that after emergence of new target and disappearance of existing target at around time $k=10$ and $k=16$, the estimated number of targets cannot follow such increase or decrease immediately. Nevertheless, after a few time steps, the estimation become precise again.

Figure 8 shows the estimated posterior PHD and original reflection signal at some time instances. Colour lines show the PHD corresponding to each target. Red line shows the label variable $l=1$, yellow $l=2$, purple $l=3$, yellow green $l=4$, and blue $l=0$. Their densities are shown in left-hand side axis. Also the black line shows the intensity of right-hand side axis. Since the peak of reflection intensity corresponds to peak of PHD, we can conclude that the estimation of position of each target is also almost accurate. Especially the increase and decrease of the number of targets are estimated accurately at time $k=6$ and $k=8$.

Figure 9 shows the expected and true value of the position of targets, $(x, h)$. Residuals of estimated position are within $20[\mathrm{~cm}]$ for 29 targets of 56 , and $50[\mathrm{~cm}]$ for 39 targets. Some false detections are shown in Figure 9. For example, one target is incorrectly detected as two targets at $k=10$, and two targets as only one target at $k=16$. They will be solved by more accurate observation model and labelling. Precise analysis on the shape of waveform corresponding to existence of targets will be the next step to find such models. 


\section{ACKNOWLEDGEMENTS}

The authors would like to thank Aero Asahi Corporation for providing LiDAR data used in this paper.

\section{REFERENCES}

Hurtt, G. C., Dubayah, R., Drake, J., Moorcroft, P. R., Pacala, S. W., Blair, J. B. and Fearon, M. G., 2004. Beyond potential vegetation: combining lidar data and a height-structured model for carbon studies. Ecological Applications, 14, pp.873883.

Ikoma, N., Yamaguchi, R., Kawano, H. and Maeda, H., 2008. Tracking of Multiple Moving Objects in Dynamic Image of Omni-Directional Camera using PHD Filter. Journal of Advanced Computational Intelligence and Intelligent Informatics, 12(1), pp.16-25.

Ikoma, N., Hasegawa, H. and Haraguchi, Y., 2013. MultiTarget Tracking in Video by SMC-PHD Filter with Elimination of Other Targets and State Dependent Multi-Modal Likelihoods. 16th International Conference on Information Fusion, pp.588595.

Kleinherenbrink, M., Ditmar, P. G. and Lindenbergh, R. C., 2014. Retracking Cryosat Data in the SARIn Mode and Robust Lake Level Extraction. Remote Sensing of Environment, 152, pp.38-50.

Mahler, R. P., 2000. A Theoretical Foundation for the SteinWinter "Probability Hypothesis Density (PHD)" Multitarget Tracking Approach. Army Research Office, Alexandria VA.

Mahler, R. P., 2003. Multitarget Bayes Filtering via First-Order Multitarget Moments. IEEE Transactions on Aerospace and Electronic Systems, 39(4), pp.1152-1178.

Nakano, K. and Chikatsu, H., 2015. On Ground Surface Extraction Using Full-waveform Airborne Laser Scanner for CIM. ISPRS Archives, 45(4/W5), pp.159-163.

Persson, A., Soderman, U., Topel, J. and Ahlberg, S., 2005. Visualization and Analysis of Full-waveform Airborne Laser Scanner Data. ISPRS Archives, 36(3/W19), pp.103-108.

Pirotti, F., 2011. Analysis of full-waveform LiDAR Data for Forestry Applications: a review of investigations and methods. iForest - Biogeosciences and Forestry, 4(3), pp.100-106.

Richter, K., Stelling, N. and Maas, H. G., 2014. Correcting Attenuation Effects Caused by Interactions in the Forest Canopy in Full-waveform Airborne Laser Scanner Data, ISPRS Archives, 40(3), pp.273-280.

Tobias, M. and Lanterman, A. D., 2004. A Probability Hypothesis Density-based Multitarget Tracker Using Multiple Bistatic Range and Velocity Measurements. IEEE Proceedings of the Thirty-Sixth Southeastern Symposium on System Theory 2004, pp.205-209.

Vo, B. N., Singh, S. and Doucet, A., 2003. Sequential Monte Carlo Implementation of the PHD Filter for Multi-target
Tracking. Proceedings of International Conference on Information Fusion, pp.792-799.

Vo, B. N., Singh, S. and Doucet, A., 2005. Sequential Monte Carlo Methods for Multi-Target Filtering with Random Finite Sets. IEEE Transactions on Aerospace and Electronic Systems, 41(4), pp.1224-1245.

Wagner, W., Ullrich, A., Ducic, V., Melzer, T. and Studnicka, N., 2006. Gaussian Decomposition and Calibration of a Novel Small-footprint Full-waveform Digitising Airborne Laser Scanner. ISPRS Journal of Photogrammetry and Remote Sensing, 60(2), pp.100-112.

Zajic, T. and Mahler, R. P., 2003. A Particle-systems Implementation of the PHD Multitarget-tracking Filter, Proceedings of SPIE 5096, Signal Processing, Sensor Fusion, and Target Recognition XII, pp.291-299. 